\title{
CauseMap: Fast inference of causality from complex time series
}

M. Cyrus Maher, Ryan D. Hernandez

Background: Establishing health-related causal relationships is a central pursuit in biomedical research. Yet, the interdependent non-linearity of biological systems renders causal dynamics laborious and at times impractical to disentangle. This pursuit is further impeded by the dearth of time series that are sufficiently long to observe and understand recurrent patterns of flux. However, as data generation costs plummet and technologies like wearable devices democratize data collection, we anticipate a coming surge in the availability of biomedically-relevant time series data. Given the life-saving potential of these burgeoning resources, it is critical to invest in the development of open source software tools that are capable of drawing meaningful insight from vast amounts of time series data.

Results: Here we present CauseMap, the first open source implementation of convergent cross mapping (CCM), a method for establishing causality from long time series data (> $\sim 25$ observations). Compared to existing time series methods, CCM has the advantage of being model-free and robust to unmeasured confounding that could otherwise induce spurious associations. CCM builds on Takens' Theorem, a well-established result from dynamical systems theory that requires only mild assumptions. This theorem allows us to reconstruct high dimensional system dynamics using a time series of only a single variable. These reconstructions can be thought of as shadows of the true causal system. If the reconstructed shadows can predict points from the opposing time series, we can infer that the corresponding variables are providing views of the same causal system, and so are causally related. Unlike traditional metrics, this test can establish the directionality of causation, even in the presence of feedback loops. Furthermore, since CCM can extract causal relationships from times series of, e.g. a single individual, it may be a valuable tool to personalized medicine. We implement CCM in Julia, a high-performance programming language designed for facile technical computing. Our software package, CauseMap, is platform-independent and freely available as an official Julia package.

Conclusions: CauseMap is an efficient implementation of a state-of-the-art algorithm for detecting causality from time series data. We believe this tool will be a valuable resource for biomedical research and personalized medicine. 


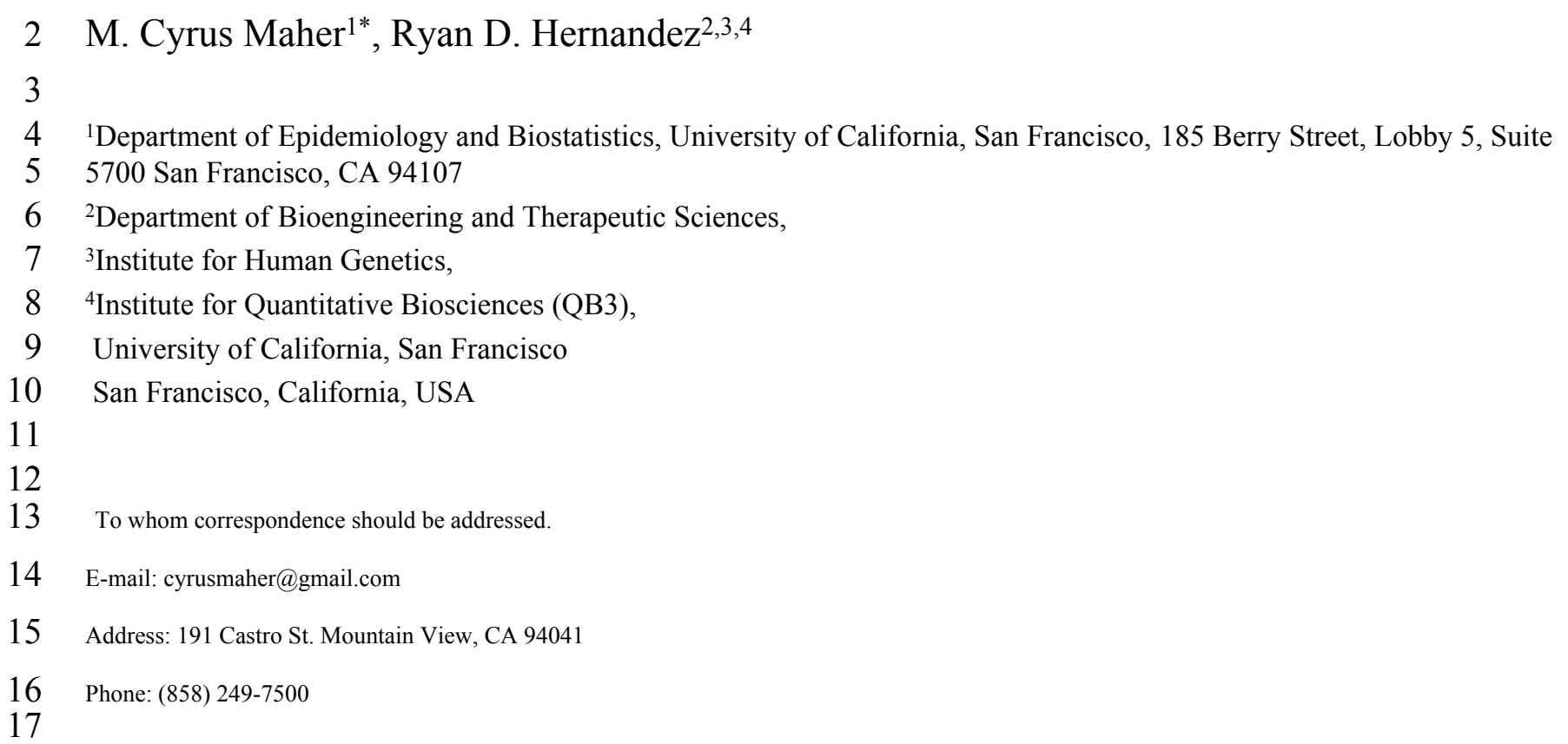




\section{Introduction}

19 Establishing health-related causal relationships is a pivotal objective in biomedical research. Yet, the

20 interdependent non-linearity of biological systems often impedes a thorough understanding of causal

21 dynamics. Existing and forthcoming time series data will likely play an important role in taming this

22 complexity. Traditional cross-sectional sampling have the limitation that they may average out non-

23 linear patterns by pooling heterogeneous signals across subjects. Long time series from a single source,

24 on the other hand, can allow us to understand dynamic and context-specific patterns of change.

We are just beginning to grasp the biomedical relevance of such a dynamical systems

26 perspective. Consider for example the human microbiome. Dysbiosis in the gut has been implicated in,

27 e.g. irritable bowel disease (IBD), obesity, diabetes, asthma, anxiety, and depression (Foster \& McVey

28 Neufeld, 2013; Arrieta et al., 2014). Meanwhile, recent studies on microbiome dynamics have found

29 that the ecological makeup of the human microbiome is dynamic and individual-specific . These

30 dynamics may also interact with pathogens in interesting and therapeutically important ways. For

31 example, there is evidence that ecological time series dynamics within the body may play a role in the 32 progression from HIV to AIDS (Vujkovic-Cvijin et al., 2013).

Complex, dynamically evolving interdependent systems such as the microbiome pose a

34 significant challenge to existing time series methods. Several metrics exist for detecting static non-

35 linear relationships. These include: Spearman rank correlation, (Spearman, 1904), distance correlation

36 (Székely \& Rizzo, 2009), and mutual information content (Kullback \& Leibler, 1951). Causal

37 relationships, on the other hand, can be examined using methods such as time-lagged regression ,

38 instrumental variables, and dynamical Bayesian networks (Granger, 1969). 
These causal methods are heavily model-based, however. As a result, they often falter when examining arbitrary non-linear or context-dependent relationships. Furthermore, the approaches

41 mentioned above cannot adequately handle feedback loops, and they frequently generate both false

42 positives and false negatives due to the influence of unmeasured confounders (Sugihara et al., 2012).

43 These are significant liabilities, particularly in biomedicine, where relationships are usually embedded 44 within a broad network of incompletely observed interactions.

In this paper, we present the first publicly available, open source implementation of convergent cross mapping (CCM), a model-free approach to detecting dependencies and inferring causality in complex non-linear systems (even in the presence of feedback loops and unmeasured confounding;

48 Sugihara et al., 2012). CCM derives this power from explicitly capturing time-dependent dynamics

49 through a technique known as state-space reconstruction (SSR). SSR has demonstrated utility for 50 problems as diverse as wildlife management and cerebral autoregulation (Vanderweele \& Arah, 2011).

51 In practice, this analysis typically requires at least 25 data points, measured with relatively high 52 accuracy and with sufficient density to capture system dynamics. One benefit of this approach is that, 53 unlike most causal inference methods, the performance of CCM improves for increasingly non-linear 54 systems. In addition, CCM can properly disentangle causal relationships that involve feedback loops, 55 provided that strong forcing from external variables does not overwhelm the dynamics of the 56 relationships of interest.

CCM leverages the fact that time series can be viewed as projections of higher-dimensional system 58 dynamics (Sugihara et al., 2012). As a logical result of this property, the time series of individual 59 variables must contain information about the full causal system. Causal dynamics (conceptualized as 60 the state space, or manifold) can then be reconstructed using individual time series. These 61 reconstructions can be thought of as shadows of the true causal system. If the shadows reconstructed 
62 from distinct variables can be used to predict points from each other's time series, we can infer that

63 these variables provide views of the same causal system and so are causally related. Since these

64 relationships are fundamentally asymmetric, this test can also establish the directionality of causation.

65 Further details on CCM are available in the supplementary material of this paper, as well as in that

66 of Sugihara et al. 2012. Additional explanatory resources can also be accessed through the project

67 website (http://cyrusmaher.github.io/CauseMap.jl).

\section{Materials and Methods}

\section{Convergent cross mapping algorithm}

70 Consider time series of hypothetical variables $X$ and $Y$. Convergent cross mapping (CCM) employs

71 time-lagged coordinates of each of these variables to produce shadow versions of their respective 72 source manifolds. To illustrate, suppose the time series for $X$ were $\{1,2,3,4\}$. Reconstructing a two-

73 dimensional shadow manifold for $X$ using a time lag of one would yield the following path: $(2,1) \rightarrow(3$,

74 2) $\rightarrow(4,3)$. For sufficiently long time series, the path of this shadow manifold is expected to reveal 75 important properties of the full causal system.

We will refer to the shadow manifolds reconstructed from $X$ and $Y$ as $M_{x}$ and $M_{y}$, respectively.

77 To test whether $X$ causes $Y$, CCM applies the following logic: because manifold reconstruction

78 preserves important structural components of the original system (i.e. the Lyapunov exponents;

79 Casdagli, Eubank, Farmer, \& Gibson, 1991), if $X$ causes $Y$, then time points that are close in $M_{y}$ should

80 also be close in $M_{x}$. Since $M_{x}$ is constructed from lags of the observations of $X$, the points that are close

81 in $M_{x}$ will also have similar values in the corresponding time series. Therefore, if $X$ causes $Y$, then $M_{y}$

82 can tell us which observations of $X$ should best predict a given held-out point from $X$. Furthermore, 
83 predictability should increase with the number of manifold points in $M_{y}$ that are considered. 84 Assessing predictive skill

To test whether $X$ causes $Y, M_{y}$ is used to infer the points in $X$ that will best predict a given

86 held-out point from $\mathrm{X}$. We measure this performance using predictive skill, quantified by $\rho_{\mathrm{ccm}}$ as

87 follows. To begin, we withhold a point from $X$ that we will then attempt to predict. We use $M_{y}$ to infer

88 the points in $M_{x}$ that will be closest to this point of interest. This is accomplished using relative

89 pairwise distances of corresponding points in $M_{y}$. We then perform a weighted average of the

90 corresponding observations in $X$ using exponential weights derived from these pairwise distances in $M_{y}$.

91 We similarly produce predicted values for each held-out point in $X . \rho_{\mathrm{ccm}}$ is then calculated as the

92 Pearson correlation between held-out and predicted points. The cross validated nature of this measure

93 serves to reduce over-fitting with respect to the model's tuning parameters described below. To

94 examine whether the signal converges as expected for a causal relationship, these steps are repeated

95 using increasing numbers of points from $M_{y}$ and $M_{x}$.

\section{CauseMap is fast}

97 CauseMap implements CCM in Julia, a high-performance programming language designed for facile 98 technical computing. Via intelligent JIT (just in time) compilation, Julia offers much of the speed of

99 low-level, low-productivity languages like $\mathrm{C}$, while also providing the ease of use and platform 100 independence of much slower high-level languages like Python, R, or Matlab.

At the core of CauseMap is the calculation of distances between a large number of manifold

102 points in potentially high dimensional spaces. To optimize efficiency, CauseMap precomputes all 103 necessary manifolds and pairwise distances using a state-of-the-art, BLAS-based protocol (for 104 benchmarks, see: https://github.com/JuliaStats/Distance.jl). 
106 runtimes for successive catenations of the time series presented in Figure 1. For our time series of

107 length 71, CauseMap finishes in approximately 10 seconds. For a time series of over 400 observations,

108 CauseMap still finishes in less than 20 minutes on a single CPU. Note that for this dataset, predictive

109 skill was nearly perfect at a time series length of 213 . This calculation finished in less than two minutes.

110 Through this example we observe that CauseMap can reach superb levels of performance long before

111 increasing time series length generates significant computational challenge.

112

\begin{tabular}{|c|c|}
\hline Time series length & Runtime (s) \\
71 & 10.2 \\
142 & 40.4 \\
213 & 116.6 \\
284 & 317.2 \\
355 & 534.7 \\
426 & 1080.5 \\
\hline
\end{tabular}

113 Table 1. Runtime versus time series length. Results are presented for one to six catenations of the dataset presented in

114 Figure 1. Runtime values are for comprehensive parameter optimizations on a single $2.6 \mathrm{GHz}$ Intel Core i7 processor,

\section{Tuning parameter values aid causal interpretation}

116 Beyond the speed and comparative simplicity resulting from cutting-edge JIT compilation, CauseMap

117 offers a number of conveniences and performance enhancements. For CCM, it is particularly important

118 to optimize two tuning parameters: $E$ and $\tau_{\mathrm{p}}$.

$E$ is the number of dimensions of the reconstructed shadow manifold. If $E_{\max }$ is the optimal

120 embedding dimension, Whitney's Theorem tells us that the dimensionality of the full causal system is

121 generically between $\left(E_{\max }-1\right) / 2$ and $E_{\max }$, inclusive (Eelles \& Toledo, 1992; Deyle \& Sugihara, 2011). 
122 Note though that $E_{\max }$ is usually unknown and must be inferred from the data. This procedure is 123 described in the following section.

$124 \tau_{\mathrm{p}}$ denotes the time delay of the causal effect of interest. By examining the optimal values of 125 these two parameters, we may place bounds on the number of variables involved in the full causal 126 system, gain insight into the timeframe of causal effects, and obtain a built-in sensitivity analysis of the 127 final results. The estimation of these parameters is described below.

\section{CauseMap optimizes and visualizes tuning parameters}

$129 E$ and $\tau_{\mathrm{p}}$ are optimized by multiple iterations of cyclic coordinate descent. This process chooses the 130 values of $E$ and $\tau_{\mathrm{p}}$ that optimize the predictive skill of the model for held-out data points. Typically 131 convergence of the cross map signal as a function of the time series length $(L)$ alone is taken as the 132 practical criterion for causality. However, measuring the dependence of this signal on $E$ and $\tau_{\mathrm{p}}$ is also 133 useful for evaluating whether the result is suitably specific with respect to the assumed structure of the 134 causal system. CauseMap therefore also includes a plotting function to visualize the dependence of the 135 predictive skill $\left(\rho_{\mathrm{ccm}}\right)$ on $\mathrm{L}$, as well as on the joint values of $\mathrm{E}$ and $\tau_{\mathrm{p}}$.

\section{Interpretation of output}

137 The systematic increase of predictive skill $\left(\rho_{\mathrm{ccm}}\right)$ with $\mathrm{L}$ constitutes a practical, qualitative criterion for 138 causality (Sugihara et al., 2012). Generally, non-causal $\rho_{\text {ccm }}$ curves are flat with respect to L, while $\rho_{\text {ccm }}$ 139 signals associated with causal signals show striking convergence given sufficient data. One exception 140 is in the case of strong external forcing. An outside variable can introduce a cross map correlation 141 between two quantities if it exerts a sufficiently strong influence over both. We speculate that such 142 situations can produce $\rho_{\mathrm{ccm}}$ values that, compared to true causal relationships, have a noisier or less 143 interpretable dependence on $\mathrm{E}$ and $\tau_{\mathrm{p} .}$. Furthermore, it is necessary to inspect the dependence of the 
144 cross map correlation on the joint distribution of $\mathrm{E}$ and $\tau_{\mathrm{p}}$ in order to properly understand the meaning

145 of the maximal values of these two variables. Note that for high throughput analyses, convergence with

146 respect to $L$ and sensitivity to $E$ and $\tau_{\mathrm{p}}$ could be assessed with, e.g. relative difference- and entropy-

147 based measures, respectively.

\section{CauseMap is easy to use}

149 Beyond the tuning parameters mentioned above, CCM requires one to specify a range of library sizes, 150 as well as the window of time points for which cross mapping should be performed. Valid values for

151 these parameters depend in turn on $E$ and $\tau_{\mathrm{p}}$. To reduce complexity for the user, CauseMap calculates 152 intelligent defaults for these parameters, while also offering the option of specifying them directly.

\section{Caveats and considerations}

154 The strengths and weaknesses of CCM make it nicely complementary to the existing tools for causal

155 inference. Unlike most algorithms for this task, the performance of CCM improves for increasingly

156 non-linear systems. This capacity depends upon relatively long time series, however. CCM requires at 157 least 25 data points, measured with relatively high accuracy and with sufficient density to capture 158 system dynamics.

159 There are also theoretical and practical limitations to the types of relationships that CCM can

160 disentangle. For example, if both $\mathrm{X}$ and $\mathrm{Y}$ are almost entirely determined by a third variable $\mathrm{Z}$, we

161 would be at risk of inferring a spurious relationship between $\mathrm{X}$ and $\mathrm{Y}$ (as we would be with any other

162 causal inference method). If the forcing from $\mathrm{Z}$ is relatively weak however, $\mathrm{CCM}$ is expected to

163 provide a lower false positive rate relative to other methods (Sugihara et al., 2012).

$164 \mathrm{CCM}$ examines relationships between variables in a pairwise fashion. However, by leveraging

165 dynamical systems theory, it has the ability to measure possibly bidirectional causal effects even in the

166 presence of unmeasured confounding. Finally, CCM performs best with complete data sampled at 
167 regular intervals. This is particularly important for inferring the time lag of the causal effect. This

168 limitation can be partially addressed through filtering or appropriate interpolation of input data.

169

\section{Results and Discussion}

171 To demonstrate CauseMap's functionality and performance, we examined the predator-prey 172 relationship between Paramecium aurelia and Didinium nasutum (Heskamp et al., 2013). Observations 173 were collected every 12 hours for 30 days, yielding a total of 60 data points (Veilleux, 1976). Plotted in

174 Figure 1 is the CauseMap visualization of the dependence of predictive skill $\left(\rho_{\mathrm{ccm}}\right)$ on $L, E$, and $\tau_{\mathrm{p}}$. In

175 Figure 1A, we observe convergence in $\rho_{\mathrm{ccm}}$ with respect to $L$, the number of data points used for 176 prediction of held-out observations. This convergence is a practical criterion for causality and the 177 source of the name convergent cross mapping.

The interpretation of this result is that the causal relationship between $P$. aurelia and $D$.

179 nasutum is bi-directional. That is, the number of predators influences the number of prey, and vice-

180 versa. Furthermore, relative strengths of convergence indicate that the top-down influence of the 181 predator (D. nasutum) is stronger than the bottom-up influence of the prey (P. Aurelia). As pointed out 182 by Sugihara et al., this finding is consistent with experimental results and illustrates the ability of CCM 183 to investigate asymmetrical bi-directional coupling in non-linear systems.

Figures $1 \mathrm{~B}$ and $1 \mathrm{C}$ show the dependence of the max $\rho_{\mathrm{ccm}}$ on $\mathrm{E}$ (the dimensionality of the reconstructed system), and the supposed time lag of the causal effect $\left(\tau_{\mathrm{p}}\right)$. Overall, the patterning of

186 these heatmaps demonstrates that $\max \rho_{\mathrm{ccm}}$ has a reasonable and moderately specific dependence on the 187 dimensionality of the reconstructed system $(E)$ and on the time lag of the causal effect $\left(\tau_{\mathrm{p}}\right)$. We expect 188 this built-in sensitivity analysis to rule out some cases of spurious convergent signal caused by external 
189 forcing. In addition, this analysis can alert the researcher when alternative combinations of $E$ and $\tau_{\mathrm{p}}$ 190 explain the data approximately as well as the optimal values of $E$ and $\tau_{\mathrm{p}}$.

191 For the system presented in Figure 1, while the $\max \rho_{\mathrm{ccm}}$ is relatively insensitive to the assumed 192 dimensionality, the best-performing $\tau_{\mathrm{p}}$ values correspond to either immediate causal effects, or those 193 delayed by five days. Note that $\tau_{\mathrm{p}}=5$ corresponds to the principal frequency of the Paramecium aurelia 194 and Didinium nasutum time series, as determined by Fourier transform analysis (see supplemental 195 materials for further details). This suggests that the peak at $\tau_{\mathrm{p}}=5$ is artifactual. Therefore, we are able to 196 infer from the data that, as we would expect, predator and prey populations exert bidirectional effects 197 in real-time.

199 Performance

200 Approximately $100 \mathrm{CCM}$ evaluations were conducted to produce Figure 1 . These calculations finished 201 in approximately 10 seconds on a single $2.6 \mathrm{GHz}$ processor. Each of these evaluations involved the 202 prediction of over 60,000 points, compiled across all sliding windows of libraries of varying lengths. 203 At an average of 1.7 microseconds per prediction, this is a highly efficient implementation given the 204 computational challenges.

\section{Dependence of predictive skill on time series length}

206 CauseMap is designed to examine causal relationships in time series with 25 or more observations. In 207 order to illustrate the effects of shorter time series, we thinned the Paramedium-Didinium data set by 208 one-half and by one-third, yielding series of 30 and 20 observations, respectively. Figure 2

209 demonstrates the effect of this reduction on the convergence of predictive skill $\left(\rho_{\mathrm{ccm}}\right)$. We see that the $2101 / 2$ thinned data set recapitulates the trends observed in the full series, including the relative 
211 magnitudes of $\rho_{\mathrm{ccm}}$ between the mappings of Didinium to Paramecium and vice versa. The $1 / 3$ thinned

212 sample set, on the other hand, no longer demonstrates convergence. In addition, compared to the longer

213 sets, it exhibits the opposite trend in relative predictive skill between the two mappings. Patterns in max

$214 \rho_{\mathrm{ccm}}$ versus $\mathrm{E}$ and $\tau_{\mathrm{p}}$ are approximately conserved, however (fig. S1).

215 This example illustrates that CCM performance drops off sharply between 20 and 30 data

216 points. This behavior is partially due to the fact that the predictive skill for a given library size is 217 averaged across sliding windows of that size. As time series get shorter, there are fewer windows of 218 appropriate size across which to average, so the estimate for predictive skill becomes much less 219 reliable.

\section{Potential biomedical applications}

221 Despite its requirement for relatively long time series ( $>25$ observations), CauseMap has the advantage 222 of requiring only a single time series for each variable. In dynamical systems with widely varying or 223 context-specific behavior, this would allow researchers to draw conclusions that are tailored to, e.g. a 224 given patient. Rather than acting on population averages, biomedical researchers would be free to fully 225 personalize therapy to the unique biology and ecology of the patient. One example of this is in the 226 treatment of microbiome dysbiosis. Imbalances in the microbiome have been implicated in, e.g. 227 irritable bowel disease (IBD), obesity, diabetes, asthma, anxiety, and depression . While fecal 228 transplantation therapy is effective in treating specific types of dysbiosis, next generation therapeutics 229 may offer a blend of purified strains, tailored to the gut ecology of the patient. We believe CauseMap 230 has the potential to be a valuable tool for designing such breakthrough therapies.

231 Additional examples include understanding patient-to-patient variability in drug response using 232 time series metabolomics, and examining the basis of e.g. influenza seasonality using global time series. 
233 We expect that such applications will continue to proliferate as the costs of data collection decrease

234 over the coming years. For this reason, we believe it is vitally important that the biomedical research

235 community have access to an efficient implementation of CCM that is user-friendly and available for

236 immediate field testing.

\section{Planned future development}

238 In future versions, we will include S-map calculations to evaluate the non-linearity of the causal system .

239 We will also add a bootstrap-based procedure for library selection, as opposed to the current approach

240 using sliding windows. This has been shown to reduce the effect of secular trends on the cross map

241 correlation (Hao Ye, George Sugihara, personal communication). In addition, we will re-implement the

242 plotting functionality in Julia, removing the requirements of Python and matplotlib for visualization.

243 Finally, we will design Python and R wrappers for CauseMap functions so that our codebase can be

244 easily leveraged from those environments as well. User suggestions will also be considered as we 245 decide how best to develop the tool.

\section{Conclusions}

247 CauseMap provides a fast, user-friendly implementation of CCM, a powerful new method for 248 exploring dependencies and even establishing causality in complex, highly non-linear datasets with 249 many unobserved variables. We believe that CCM holds a great deal of promise for a wide range of 250 applications, including personalized microbiome therapy and metabolic dynamics analysis. As novel 251 time series datasets continue to emerge, it is our hope that CauseMap will allow researchers to uncover 252 interesting and biomedically actionable causal relationships using this next-generation time series 253 method. 


\section{Availability and Requirements}

256 Project name: CauseMap

257 Project home page: http://cyrusmaher.github.io/CauseMap.j1/

258 Operating system(s): Platform independent

259 Programming language: Julia

260 Other requirements: Python and matplotlib (for graphing)

261 License: MIT

262 Any restrictions to use by non-academics: No

\section{List of abbreviations}

264 Convergent cross mapping (CCM), State space reconstruction (SSR)

\section{Competing interests}

266 The authors had no competing interest to declare.

\section{Author's contributions}

$268 \mathrm{MCM}$ and $\mathrm{RDH}$ conceived the project and drafted the manuscript. MCM implemented the algorithm 269 and built the project website.

\section{Author's Information}

271 M. C. M. is a University of California, San Francisco (UCSF) graduate student with an emphasis in

272 statistical computing. After graduation, he will be working as a Software Engineer for Human

273 Longevity Inc., a San Diego-based biotechnology startup. R. D. H. is a Bioengineering \& Therapeutics

274 Sciences professor at UCSF. He is also the author of SFS_CODE a popular program for flexible

275 simulation of population genetic evolution. 


\section{Acknowledgements}

277 We would like to thank George Sugihara, Hao Ye, and Ethan Deyle for their invaluable help in

278 understanding the core details of the CCM algorithm, and Lawrence Uricchio, Nicolas Strauli, and 279 Raul Torres for comments on this manuscript. 


\section{REFERENCES}

281

282

283

284

Arrieta M-C, Stiemsma LT, Amenyogbe N, Brown EM, Finlay B. 2014. The Intestinal Microbiome in Early Life: Health and Disease. Frontiers in Immunology 5:427.

Casdagli M, Eubank S, Farmer JD, Gibson J. 1991. State space reconstruction in the presence of noise. Physica D: Nonlinear Phenomena 51:52-98.

Deyle ER, Sugihara G. 2011. Generalized theorems for nonlinear state space reconstruction. PloS one 6:e18295.

Eelles J, Toledo D. 1992. Collected Papers of Hassler Whitney. Boston: Birkhauser Publications.

Foster JA, McVey Neufeld K-A. 2013. Gut-brain axis: how the microbiome influences anxiety and depression. Trends in neurosciences 36:305-12.

Granger CWJ. 1969. Investigating Causal Relations by Econometric Models and Cross-spectral Methods Title. Econometrica 37:424-438.

Heskamp L, Meel-van den Abeelen A, Katsogridakis E, Panerai R, Simpson D, Lagro J, Claassen J. 2013. Convergent cross mapping: a promising technique for future cerebral autoregulation estimation. CEREBROVASCULAR DISEASES 35:15-16.

Kullback S, Leibler RA. 1951. On Information and Sufficiency. The Annals of Mathematical Statistics 22:79-86.

Spearman C. 1904. The proof and measurement of association between two things. American Journal of Psychology 15:72-101.

Sugihara G, May R, Ye H, Hsieh C, Deyle E, Fogarty M, Munch S. 2012. Detecting causality in complex ecosystems. Science (New York, N.Y.) 338:496-500.

Székely GJ, Rizzo ML. 2009. Brownian distance covariance. The Annals of Applied Statistics 3:12361265.

Vanderweele TJ, Arah OA. 2011. Bias formulas for sensitivity analysis of unmeasured confounding for general outcomes, treatments, and confounders. Epidemiology (Cambridge, Mass.) 22:42-52.

Veilleux BG. 1976. The analysis of a predatory interaction between Didinium and Paramecium. Master's thesis, University of Alberta.

Vujkovic-Cvijin I, Dunham RM, Iwai S, Maher MC, Albright RG, Broadhurst MJ, Hernandez RD, Lederman MM, Huang Y, Somsouk M et al. 2013. Dysbiosis of the gut microbiota is associated with HIV disease progression and tryptophan catabolism. Science translational medicine 5:193ra91. 


\section{Figure legends}

314 Fig. 1. An example visualization from CauseMap using abundances of Paramecium aurelia and Didinium nasutum

315 (see supplemental materials for more information on this system). A.) For optimal parameter values, the convergence of the 316 cross-map correlation with library size. B-C.) The dependence of the maximum cross-map correlation on assumed dimensionality (measured by E) and the time lag of the causal effect (measured by $\tau_{\mathrm{p}}$ ). Note that the second maximum at

$318 \tau_{\mathrm{p}}=5$ corresponds to the principal frequency of the $P$. aurelia and $D$. nasutum time series, as determined by Fourier 319 transform analysis.

Fig. 2. The effect of time series length on $\rho_{\text {ccm }}$ convergence. Black, blue, and red lines illustrate $\rho_{\mathrm{ccm}}$ for the full, $1 / 2$ thinned, and 1/3 thinned datasets, respectively. For a given color, darker lines show $\rho_{\mathrm{ccm}}$ for the test of whether Didinium

323 abundance influences Paramecium abundance. Lighter lines examine the converse. 


\section{1}

An example visualization from CauseMap using abundances of Paramecium aurelia and Didinium nasutum

See supplemental materials for more information on this system. A.) For optimal parameter values, the convergence of the cross-map correlation with library size. B-C.) The dependence of the maximum cross-map correlation on assumed dimensionality (measured by E) and the time lag of the causal effect (measured by $\tau_{p}$ ). Note that the second maximum at $\tau_{p}=5$ corresponds to the principal frequency of the $P$. aurelia and $D$. nasutum time series, as determined by fourier transform analysis.

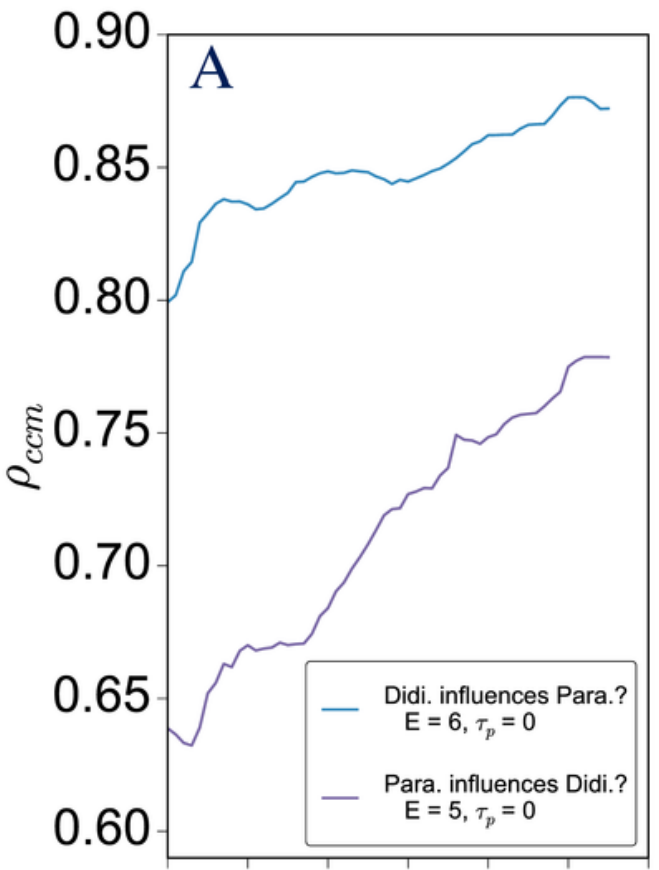

10203040506070
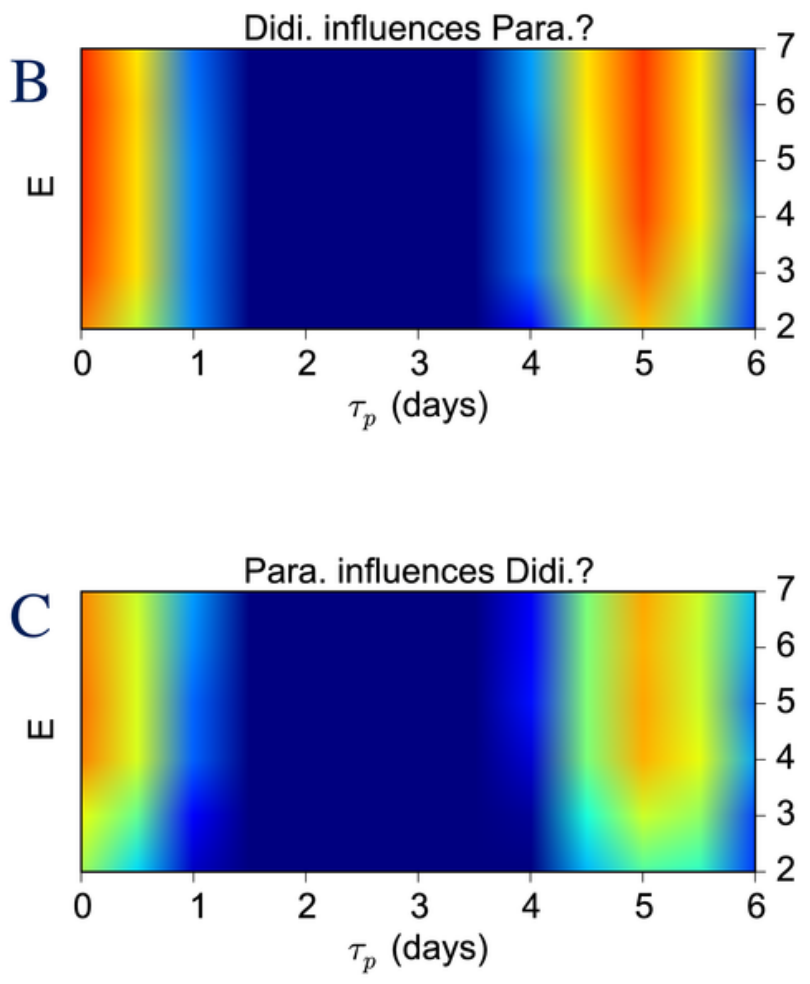

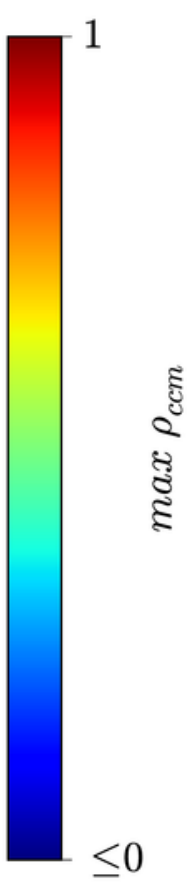


2

The effect of time series length on $\rho_{c c m}$ convergence.

Black, blue, and red lines illustrate $\boldsymbol{\rho}_{\mathrm{ccm}}$ for the full, $1 / 2$ thinned, and 1/3 thinned datasets, respectively. For a given color, darker lines show $\rho_{\text {ccm }}$ for the test of whether Didinium abundance influences Paramecium abundance. Lighter lines examine the converse.

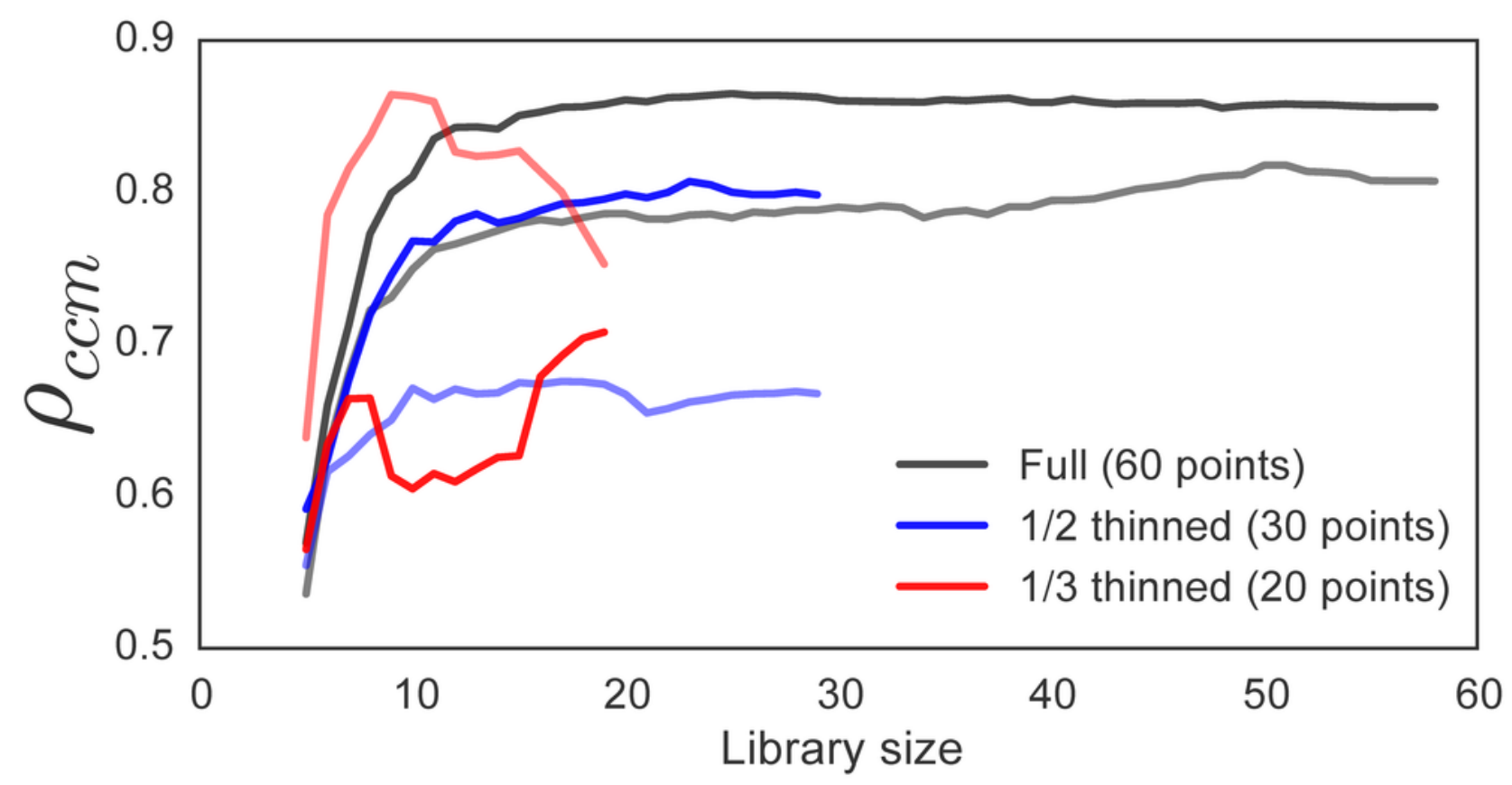




\section{Table $\mathbf{1}$ (on next page)}

Table 1. Runtime versus time series length.

Results are presented for one to six catenations of the dataset presented in Figure 1.

Runtime values are for comprehensive parameter optimizations on a single $2.6 \mathrm{GHz}$ Intel Core i7 processor 


\begin{tabular}{|c|c|}
\hline Time series length & Runtime (s) \\
\hline 71 & 10.2 \\
\hline 142 & 40.4 \\
\hline 213 & 116.6 \\
\hline 284 & 317.2 \\
\hline 355 & 534.7 \\
\hline 426 & 1080.5 \\
\hline
\end{tabular}

2

3

4

5 Table 1. Runtime versus time series length. Results are presented for one to six catenations of the dataset presented in

6 Figure 1. Runtime values are for comprehensive parameter optimizations on a single $2.6 \mathrm{GHz}$ Intel Core i7 processor 